\title{
Critical Sets for 3D Reconstruction Using Lines
}

\author{
Thomas Buchanan \\ Eberstadt, Troyesstr. 64, D-6100 Darmstadt, Germany
}

\begin{abstract}
This paper describes the geometrical limitations of algorithms for $3 \mathrm{D}$ reconstruction which use corresponding line tokens. In addition to announcing a description of the general critical set, we analyse the configurations defeating the Liu-Huang algorithm and study the relations between these sets.
\end{abstract}

\section{Introduction}

The problem of $3 \mathrm{D}$ reconstruction is to determine the geometry of a three-dimensional scene on the basis of two-dimensional images. In computer vision it is of utmost importance to develop robust algorithms for solving this problem. It is also of importance to understand the limitations of the algorithms, which are presently available, because knowledge of such limitations guides their improvement or demonstrates their optimality.

From a theoretical point of view there are two types of limitations. The first type involves sets of images, where there exist more than one essentially distinct 3D scene, each giving rise to the images. The superfluous reconstructions in this case can be thought of as "optical illusions". This type of limitation describes the absolute "bottom line" of the problem, because it involves scenes where the most optimal algorithm breaks down.

The second type of limitation is specific to a given not necessarily optimal algorithm. It describes those scenes which "defeat" that particular algorithm.

Currently, algorithms for 3D reconstruction are of two types. One type assumes a correspondence between sets of points in the images. For algorithms of this type the critical set has been studied extensively. (See [6] for a vivid graphical description of this locus and the references in [8] for a detailed bibliography.) In recent years another type of algorithm has been introduced, which assumes a correspondence between sets of lines in the images.

The purpose of this paper is to describe limitations for the algorithms which use lines as tokens in the images.

We use projective geometry throughout the paper. Configurations in 3-space are considered to be distinct if they cannot be transformed into one another by a projective linear transformation. The use of the projective standpoint can be thought of as preliminary to studying the situation in euclidean space. But the projective situation is of interest in its own right, because some algorithms operate essentially within the projective setting. Generally, algorithms using a projective setting are easier to analyse and implement than algorithms which fully exploit the euclidean situation.

This paper is organized as follows. In section 2 we collect some standard definitions from line geometry, which will allow us to describe the line sets in Sections 3 and 4 . In Section 3 we describe line sets $\Psi$ in 3 -space and images of $\Psi$ which give rise to ambiguous reconstructions. In Section 4 we describe line sets $\Gamma$ in 3-space which defeat the algorithm introduced in [7]. Essential properties of $\Gamma$ were first noted in $[10$, p. 106] in the context of constructive geometry. In Section 5 we discuss the relationship between $\Psi$ and $\Gamma$. 
A proof of Theorem 3.1 will appear in [1].

\section{Definitions from line geometry}

The set of all lines in 3-space will be denoted by $\Omega$. It is well-known that $\Omega$ is a 4 dimensional algebraic variety. (See [11, pp. 244-247 and Chap. XV] for an introduction to $\Omega$ and [15] for an encyclopedic exposition.) To see that $\operatorname{dim} \Omega=4$ is plausible, consider the set of pairs of points in 3-space. The dimension of this set is $2 \times 3=6$. Each pair of distinct points determines a line $l$ joining the two points. However, $l$ is overdetermined for we can move each of the two points along $l$. This reduces the degrees of freedom for $\Omega$ by 2 . Thus we have $\operatorname{dim} \Omega=6-2=4$.

Elements of $\Omega$ can be coordinatized by 6 -tuples $\left(p_{01}, p_{02}, p_{03}, p_{12}, p_{13}, p_{23}\right)$ which are subject to the following conditions.

(a) At least one $p_{i j}(0 \leq i<j \leq 3)$ is nonzero.

(b) Scalar multiples $\left(\lambda p_{01}, \lambda p_{02}, \lambda p_{03}, \lambda p_{12}, \lambda p_{13}, \lambda p_{23}\right)$ denote the same line for all $\lambda \neq 0$.

(c) The line coordinates $p_{i j}$ satisfy the equation

$$
p_{01} p_{23}-p_{02} p_{13}+p_{03} p_{12}=0
$$

Given a line $l$ containing distinct points with homogeneous coordinates $\left(x_{0}, \ldots, x_{3}\right)$ and $\left(y_{0}, \ldots, y_{3}\right)$, then the $p_{i j}$ are defined by

$$
p_{i j}=\operatorname{det}\left(\begin{array}{ll}
x_{i} & x_{j} \\
y_{i} & y_{j}
\end{array}\right)(0 \leq i<j \leq 3)
$$

That the $p_{i j}$ do indeed have properties (a), (b) and (c) is shown in [11] for example.

An algebraic set is defined to be a set which is defined by a set of polynomial equations. In line geometry these equations involve the line coordinates $p_{i j}$ as unknowns. An algebraic set is called reducible if it can be written as the union of two nonempty proper algebraic subsets. For example, in the cartesian plane the set of points satisfying $x y=0$, which consists of the coordinate axes, is a reducible algebraic set, because the set is the union of the $y$-axis $(x=0)$ and the $x$-axis $(y=0)$. On the other hand, the $x$-axis described by $y=0$ is irreducible, because the only proper algebraic subsets of the $x$-axis are finite sets of points of the form $(x, 0)$. An irreducible algebraic set is called a variety. It can be shown that any algebraic set can be described as the finite union of varieties.

A variety $V$ has a well-defined dimension, which is the number of parameters required to parametrize smooth open subsets of $V$. We can think of the dimension of $V$ as the number of degrees of freedom in $V$. For example, the plane has dimension 2, 3-space has dimension 3 , etc.

Line varieties $\Lambda$ are subvarieties of $\Omega$. Since $\operatorname{dim} \Omega=4$, there four possibilities for $\operatorname{dim} \Lambda$, when $\Lambda$ is not all of $\Omega$. If $\operatorname{dim} \Lambda=0$, then $\Lambda$ is a single element of $\Omega$, i.e., a line. Line varieties of dimension 1,2 and 3 are called a ruled surface, a (line) congruence and a (line) complex respectively. The unfortunate choice of terminology for line varieties goes back to the 19th century. The terms have so thoroughly established themselves in the literature, however, that it would be futile to try to introduce new names for the line varieties. 
Note that a ruled surface as defined above is a 1-parameter family of lines, not a set of points. For example, the hyperboloid of one sheet contains a 1-parameter family of lines-a ruled surface.

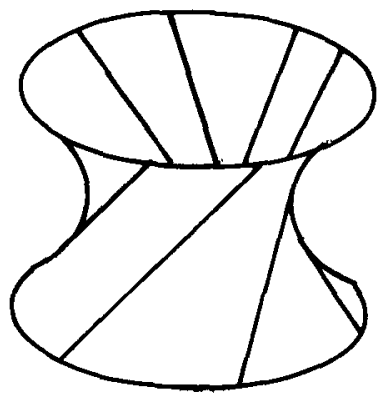

A different ruled surface lying on the hyperboloid is shown in figure below.

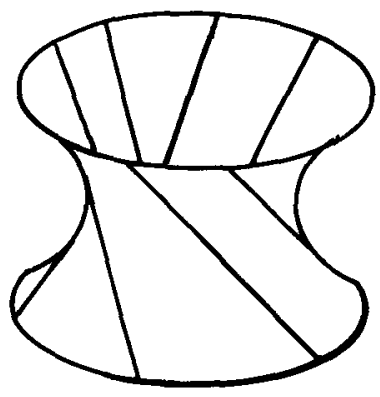

A particularly simple ruled surface is a pencil defined to be the set of lines passing through a given point $P$ and lying in a given plane $\pi$.

An important descriptor for a line complex $\Gamma$ is its order. The order of $\Gamma$ is defined to be the number of lines $\Gamma$ has in common with a general pencil. It is important to count not only lines real space but also properly count lines in the space of complex numbers. For any point $P$ in 3-space we may consider all lines of $\Gamma$ which pass through $P$. This subset of $\Gamma$ is called the complex cone at $P$. The order of $\Gamma$ could equivalently be defined as the order of a general complex cone of $\Gamma$. If a general complex cone has as its base a plane curve of degree $d$, then $d$ is the order of the cone and the order of $\Gamma$.

A theorem of Felix Klein states that in the space over the complex numbers a line complex can be described by a single homogeneous polynomial equation

$$
f\left(p_{01}, p_{02}, p_{03}, p_{12}, p_{13}, p_{23}\right)=0
$$

(see [4, p.147, Exercise 6.5d]). Of course, it is always tacitly assumed that (1) holds. If $\Gamma$ is described by a homogeneous polynomial $f$, the order of $\Gamma$ coincides with the degree of $f$.

A very simple line complex consists of all lines which meet a given line $l$. If the coordinates of $l$ are $a=\left(a_{01}, a_{02}, a_{03}, a_{12}, a_{13}, a_{23}\right)$, then the equation for this complex can be shown to be

$$
a_{01} p_{23}-a_{02} p_{13}+a_{03} p_{12}-a_{13} p_{02}+a_{12} p_{03}+a_{23} p_{01}=0 .
$$


This polymonial has degree 1 so the order of the complex is 1 . The polynomial in $a$ and $p=\left(p_{01}, p_{02}, p_{03}, p_{12}, p_{13}, p_{23}\right)$ is denoted by $\Omega_{a p}$. Equation (1), which we are always tacitly assuming, can be expressed by the equation $\Omega_{p p}=0$.

For a given complex $\Gamma$ it may happen that $\Gamma$ contains all lines through some special point $P$. In this case $\Gamma$ is called a total point of $\Gamma$.

Given a line congruence $\Psi$ only a finite number of lines pass through a given point in general. Again we count not only lines in real space but lines in the space over the complex numbers. The number of such lines is constant for almost all points of 3-space; this number is defined to be the order of $\Psi$. Analogously, a general plane $\pi$ in 3-space contains only a finite number of lines of $\Psi$. This number is defined to be the class of $\Psi$. Points lying on an infinite number of lines of $\Psi$ and planes containing an infinite number of lines of $\Psi$ are called singular.

Given a congruence $\Psi$ and a line $l$ in 3 -space not in $\Psi$, we may consider the subset of $\Psi$ consisting of elements of $\Psi$ which meet $l$. This set can then be described by the equations which define $\Psi$ together with an additional linear equation of the form of (2). If this set is irreducible, it is a ruled surface.

In general, there exist a finite number of points $P$ on $l$ with the property that $l$ together with two elements of $\Psi$ through $P$ lie in a plane. This number is the same for almost all $l$ and is defined to be the rank of $\Psi$. A congruence of order $n$, class $m$ and rank $r$ is referred to as a $(n, m, r)$-congruence.

Given a point $P$ all lines through $P$ form a $(1,0,0)$-congruence called the star at $P$.

A ruled surface $\rho$ can be considered to be an algebraic space curve in 5 dimensional projective space, which is the space coordinatized by the six homogeneous line coordinates $p_{i j}(0 \leq i<j \leq 3)$. The curve lies on the variety defined by (1). The order of $\rho$ is defined to be the number of lines which meet a general given line, where again lines are counted properly in the space of complex numbers. For example, ruled surfaces lying on a hyperboloid have order 2.

If a (space) curve in complex projective space is smooth, it is topologically equivalent (homeomorphic) to a surface (a so-called Riemann surface), which is either a sphere, a torus or a surface having a finite number of handles.

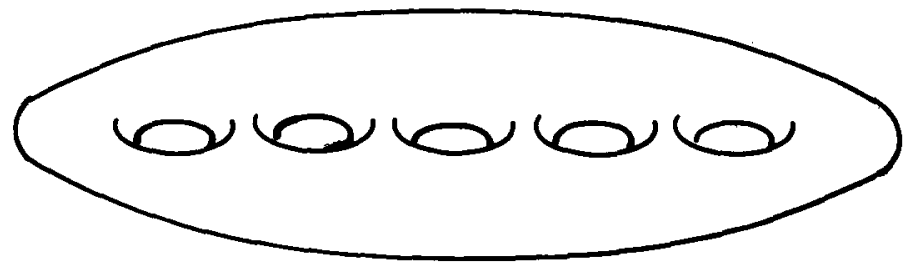

(The surface in the figure above has 5 handles.)

Surfaces with handles can be topologically built up from tori by cutting small disks out of the tori and pasting them together on the disk boundries. The number of tori required to build up a given surface is the number of handles of the surface; this number is defined to be the genus of the curve. The definition of genus can be extended to curves with singularities. We refer the reader to a textbook on algebraic curves or algebraic geometry (for example, [12] or [14]) for equivalent definitions of "genus". The concept of 
genus is applicable to ruled surfaces, since these can be regarded as space curves.

Given a congruence $\Psi$, the sectional genus of $\Psi$ is defined to be the genus of a general ruled surface $\rho$ consisting of the elements of $\Psi$ which meet a given line $l$ not lying in $\Psi$.

\section{The critical line set}

In this section we assume three cameras are set up in general position with centers $\mathrm{O}_{1}, \mathrm{O}_{2}, \mathrm{O}_{3}$. The image planes are denoted by $I_{1}, I_{2}, I_{3}$. The imaging process defines collineations $\gamma_{i}: \operatorname{star}\left(O_{i}\right) \longrightarrow I_{i}(i=1,2,3)$, which we assume to be entirely general. To consider the critical set, we consider another three centers $\bar{O}_{1}, \bar{O}_{2}, \bar{O}_{3}$, which are in general position with respect to each other and the first set of centers $O_{1}, O_{2}, O_{3}$. The symbols with bars denote an alternative reconstruction of the scene and the camera positions. The stars at the $\bar{O}_{i}$ 's project to the same image planes defining collineations $\bar{\gamma}_{i}: \operatorname{star}\left(\bar{O}_{i}\right) \longrightarrow I_{i}$, also of general type. The compositions $\alpha_{i}=\gamma_{i} \circ \bar{\gamma}_{i}^{-1}$ define collineations between the lines and the planes through $O_{i}$ and $\bar{O}_{i}$.

We shall describe what we mean by "general position" after stating our main result.

Theorem 3.1 With respect to images from three cameras the general critical set $\Psi$ for the reconstruction problem using lines is a $(3,6,5)$-congruence. The sectional genus of $\Psi$ is $5 . \Psi$ contains 10 singular points, 3 of which are located at the camera centers. The singular cones have order 9 and genus $1 . \Psi$ has no singular planes.

The proof of this theorem is given in [1]. Essentially, the proof determines $\Psi$ 's order and class and $\Psi$ 's singular points and planes. These invariants suffice to identify $\Psi$ in the classification of congruences of order 3 given in [3]. In this classification the other properties of $\Psi$ can be found.

Just as a ruled surface can be considered to be a curve in 5-space, a congruence can be considered to be a surface in 5 -space.

According to [3, p. 72] $\Psi$ is a surface of order 9 in 5 -space. This surface has a plane representation: the hyperplane sections of $\Psi$, i.e., the intersection of $\Psi$ with complexes of order 1, correspond to the system of curves of order 7 , which have nodes at 10 given base points. The plane cubic curves which pass through 9 of the 10 base points correspond to the singular cones of $\Psi$.

Let us now describe what is meant by "general position".

First, we assume the centers of projection $\mathrm{O}_{1}, \mathrm{O}_{2}, \mathrm{O}_{3}$ and $\bar{O}_{1}, \bar{O}_{2}, \bar{O}_{3}$ are not collinear. Let $\pi$ denote the plane spanned by $O_{1}, O_{2}, O_{3}$ and $\bar{\pi}$ denote the plane spanned by $\bar{O}_{1}, \bar{O}_{2}, \bar{O}_{3}$.

Next, we assume that the images of $\pi$ under the various $\alpha_{i}$ intersect in a single point $\vec{P}=\pi^{\alpha_{1}} \cap \pi^{\alpha_{2}} \cap \pi^{\alpha_{3}}$. Analogously, we assume the images of $\bar{\pi}$ under $\alpha_{1}^{-1}, \alpha_{2}^{-1}, \alpha_{3}^{-1}$ intersect in a single point $P=\bar{\pi}_{1}^{\alpha_{1}^{-1}} \cap \bar{\pi}^{\alpha_{2}^{-1}} \cap \bar{\pi}^{\alpha_{3}^{-1}}$.

Each pair of centers $O_{i}, O_{j}$ and collineations $\alpha_{i}, \alpha_{j}(i \neq j=1,2,3)$ determines a point locus $Q_{i j}$, which is critical for $3 \mathrm{D}$ reconstruction using points. In the general projective setting $Q_{i j}$ is a quadric surface passing through $O_{i}$ and $O_{j}$. We assume each $Q_{i j}$ is a proper quadric and each pair of quadrics $Q_{i j}, Q_{i k}(\{i, j, k\}=1,2,3)$ intersect in a irreducible curve of order 4 . Moreover, we assume that all three quadrics intersect in 8 distinct points. The analogous assumptions are assumed to hold for the quadrics $\bar{Q}_{i j}$ determined by the centers $\bar{O}_{i}, \bar{O}_{j}$.

Finally, we assume that for each fixed $i=1,2,3$ the two lines $\left(O_{i} O_{j}\right)^{\alpha_{j}}, j=1,2,3, j \neq$ i) are skew. Here $O_{i} O_{j}$ denotes the line joining $O_{i}$ and $O_{j}$. 


\section{Line sets defeating the Liu-Huang algorithm}

The algorithm proposed in [7] sets about to determine the rotational components of the camera orientations with respect to one another in its first step. We shall only concern ourselves with this step in what follows.

If three cameras are oriented in a manner that they differ only by a translation, we can define a collineation between the lines and planes through each center of projection $O_{i}(i=1,2,3)$ by simply translating the line or the plane from one center to the other. This collineation coincides with the collineation at the $O_{i}$ induced by the images, namely where the points $P_{i}$ and $P_{j}$ in the $i$-th and $j$-th image correspond when they have the same image coordinates $(i, j=1,2,3)$.

Regardless of camera orientation, introducing coordinates in the images preemptively determines collineations between the images and as a result between the corresponding lines and planes through the centers of projection. We call such lines and planes homologous, i.e., the images of homologous elements have the same coordinates in the various images.

In the case where the cameras are simply translated, homologous elements in the stars at $O_{i}$ are parallel. Projectively speaking, this means that homologous rays intersect in the plane at infinity and homologous planes are coaxial with the plane at infinity.

A generalization of the translational situation arises when the collineations between the lines and planes through the centers are induced by perspectivities with a common axial plane $\pi$, i.e., a ray $r_{i}$ through $O_{i}$ corresponds to $r_{j}$ through $O_{j}$ when $r_{j}=\left(r_{i} \cap\right.$ $\pi) O_{j}(i, j=1,2,3)$. Here $\left(r_{i} \cap \pi\right) O_{j}$ denotes the line joining points $r_{i} \cap \pi$ and $O_{j}$.

Note that the projections of points $X$ on $\pi$ give rise to homologous rays $O_{i} X$, which per definition have the same coordinates in the images. Let $l$ be a line in 3-space and $l_{i}(i=1,2,3)$ denote the images of $l$. If $l$ meets $\pi$ in $X$, the points $P_{i}$ corresponding to the projection of $X$ in the images have the same coordinates. (In the translation case $X$ corresponds to the vanishing point of $l$.) Thus if the $l_{i}$ are drawn in a single plane using the common coordinate system of the images, they are concurrent, because the $P_{i} \in l_{i}$ all have the same coordinates. In the translational case, this point is the vanishing point of the parallel class of $l$.

The idea behind the first step in the algorithm of [7] is to find the rotational components of the camera orientation by collinearly rearranging two of the images so that all corresponding lines in all three images are simultanously concurrent with respect to a given coordinate systems. If

$$
\sum_{i=0}^{2} u_{i} x_{i}=0, \sum_{i=0}^{2} v_{i} x_{i}=0, \sum_{i=0}^{2} w_{i} x_{i}=0
$$

are the equations of the projections of a line $l$, we look for rotations, i.e., $3 \times 3$ orthogonal matrices, or more generally simply $3 \times 3$ invertible matrices $M_{1}, M_{2}$ such that $u=$ $\left(u_{0}, u_{1}, u_{2}\right), M_{1} v=M_{1}\left(v_{0}, v_{1}, v_{2}\right)$ and $M_{2} w=M_{2}\left(w_{0}, w_{1}, w_{2}\right)$ are linearly dependent, the linear dependancy being equivalent to concurrency. This means we look for $M_{1}, M_{2}$ such that

$$
\operatorname{det}\left(u, M_{1} v, M_{2} w\right)=0
$$

for all triples of corresponding lines in the images. The algorithm would like to infer that after applying $M_{1}$ and $M_{2}$, the cameras are now oriented so that they are translates of each other, or in the projective case that the images are perspectively related by a common axial plane.

Consider the cameras with general orientations, where again homologous rays through the centers corespond to points in the image having the same coordinates. If a line $l$ in 
space meets 3 homologous rays $r_{1}, r_{2}, r_{3}$, then the projections of $l$ are concurrent, the point of concurrency being the point corresponding to $r_{1}, r_{2}$ and $r_{3}$.

The set of all lines which meet the rays $r_{1}, r_{2}$ and $r_{3}$ when the homologous rays are skew is a ruled surface of order 2 denoted by $\left[r_{1}, r_{2}, r_{3}\right]$. Let $\Gamma=\bigcup_{r_{1}, r_{2}, r_{3}}\left[r_{1}, r_{2}, r_{3}\right]$ be the set of all lines of 3 -space meeting triples of homologous rays. If all the lines in the scene lie in $\Gamma$, then their projections have the property that they are concurrent. But since the cameras were in general position, they are not translates of each other. Thus $\Gamma$ defeats the algorithm.

To find the equation for $\Gamma$ let $q_{1}, q_{2}, q_{3}$ denote the line coordinates of 3 rays through $O_{1}$, not all in a plane. Then $q_{1}, q_{2}, q_{3}$ form a frame of reference for rays through $O_{1}$; the coordinates of any ray through $O_{1}$ can be written as a nonzero linear combination

$$
\lambda_{1} q_{1}+\lambda_{2} q_{2}+\lambda_{3} q_{3}
$$

of the three coordinate 6-tuples $q_{1}, q_{2}, q_{3}$.

If $s_{1}, s_{2}, s_{3}$ denote the rays through $O_{2}$, and $t_{1}, t_{2}, t_{3}$ the rays through $O_{3}$ which are homologous to $q_{1}, q_{2}, q_{3}$, the line coordinates of the rays through $O_{2}$ and $O_{3}$, which are homologous to the ray defined by (3) are given by

$$
\lambda_{1} s_{1}+\lambda_{2} s_{2}+\lambda_{3} s_{3} \text { and } \lambda_{1} t_{1}+\lambda_{2} t_{2}+\lambda_{3} t_{3}
$$

Thus a line $l$ with coordinates $p$ intersect this homologous triple if and only if

$$
\begin{aligned}
& 0=\Omega_{p, \lambda_{1} q_{1}+\lambda_{2} q_{2}+\lambda_{3} q_{3}}=\sum_{i=1}^{3} \lambda_{i} \Omega_{p q_{i}} \\
& 0=\Omega_{p, \lambda_{1} s_{1}+\lambda_{2} s_{2}+\lambda_{3} s_{3}}=\sum_{i=1}^{3} \lambda_{i} \Omega_{p s_{i}} \\
& 0=\Omega_{p, \lambda_{1} t_{1}+\lambda_{2} t_{2}+\lambda_{3} t_{3}}=\sum_{i=1}^{3} \lambda_{i} \Omega_{p t_{i}}
\end{aligned}
$$

In general $l$ with line coordinates $p$ lies in $\Gamma$ if there exist $\left(\lambda_{1}, \lambda_{2}, \lambda_{3}\right)$ not all zero such that $p$ satisfies the equations above. This will be the case when

$$
\operatorname{det}\left(\begin{array}{lll}
\Omega_{p q_{1}} & \Omega_{p q_{2}} & \Omega_{p q_{3}} \\
\Omega_{p s_{1}} & \Omega_{p s_{2}} & \Omega_{p s_{3}} \\
\Omega_{p t_{1}} & \Omega_{p t_{2}} & \Omega_{p t_{3}}
\end{array}\right)=0
$$

Thus (4) is the equation for $\Gamma$; the left-hand side of (4) is a homogeneous polynomial in $p=\left(p_{01}, p_{02}, p_{03}, p_{12}, p_{13}, p_{23}\right)$ of degree 3 . We have the following theorem.

Theorem 4.1 The set $\Gamma$ which defeats the Liu-Huang algorithm is in general a line complex of order 3 given by (4), where $q_{1}, q_{2}, q_{3} ; s_{1}, s_{2}, s_{3}$ and $t_{1}, t_{2}, t_{3}$ denote line coordinates of rays through $\mathrm{O}_{1}, \mathrm{O}_{2}$ and $\mathrm{O}_{3}$ respectively. The centers are total points of $\Gamma$.

To prove the assertion about the total points note that if say $O_{1} \in l$ then $\Omega_{p q_{i}}=0$ for $i=1,2,3$; hence $p$ satisfies (4).

In the euclidean case (4) takes on the special form in which the triples $q_{1}, q_{2}, q_{3} ; s_{1}, s_{2}, s_{3}$ and $t_{1}, t_{2}, t_{3}$ are line coordinates for an orthogonal triple of lines through $O_{1}, O_{2}$ and $O_{3}$ respectively.

Definition: $\Gamma$ is called the complex of common transversals of homologous rays. The essential properties of $\Gamma$ were first noted in [10, p. 106] in the context of constructive geometry. The projective geometry of $\Gamma$ has also been studied in [5] and [13, IV,pp. 134 ff.]. 


\section{The relation between critical congruence $\Psi$ and the complex of common transversals $\Gamma$}

Before going into the relation between $\Gamma$ and $\Psi$ we state some properties of still another congruence.

The Roccella congruence $\Delta$ is a $(3,3,2)$-congruence of sectional genus 2 which consists of all common transversals of 3 homographically related plane pencils in general position. If we restrict the collineations of three stars to a plane pencil, we obtain $\Delta$ as a subset of the complex of common transversals determined by collinear stars. (Cf. [9], [2, pp. 152-157].)

Let us return to the situation used in defining $\Psi$. Here $\mathrm{O}_{1}, \mathrm{O}_{2}, \mathrm{O}_{3}$ and $\bar{O}_{1}, \bar{O}_{2}, \bar{O}_{3}$ denote the location of the cameras for two essentially different $3 \mathrm{D}$ reconstructions and $\alpha_{i}$ denote collineations between the stars at $O_{i}$ and $\bar{O}_{i}$, which are induced by the images.

Any plane $\bar{\pi}$ in 3-space not meeting $\bar{O}_{1}, \bar{O}_{2}, \bar{O}_{3}$ determines perspectivities between stars at $\bar{O}_{i}$ via $\bar{r}_{i} \mapsto\left(\bar{r}_{i} \cap \bar{\pi}\right) \bar{O}_{j}(i, j=1,2,3)$. Since $\operatorname{star}\left(O_{i}\right)$ and $\operatorname{star}\left(\bar{O}_{i}\right)$ are collinear via $\alpha_{i}$, these perspectivities also induce collineations between the stars at $O_{i}$. Hence $\bar{\pi}$ also gives rise to a complex $\Gamma_{*}$ of common transversals of homologous rays, as explained in the previous section.

Proposition 5.1 If $\bar{\pi}_{1}, \bar{\pi}_{2}$ are two distinct planes in 3-space in general position, then

$$
\Gamma_{\pi_{1}} \cap \Gamma_{\hbar_{2}}=\Psi \cup \Delta \cup \bigcup_{i=1,2,3} \operatorname{star}\left(O_{i}\right)
$$

where $\Delta$ denotes the Roccella congruence induced by the pencils at $O_{i}$ in the planes $\left(\bar{O}_{i}\left(\bar{\pi}_{1} \cap \bar{\pi}_{2}\right)\right)^{\alpha_{i}^{-1}}(i=1,2,3)$.

Proof. "ב". We need only show that

$$
\Psi \backslash \bigcup \operatorname{star}\left(O_{i}\right) \subseteq \Gamma_{\pi_{1}}
$$

since $\Gamma_{\pi_{1}}$ contains $\bigcup \operatorname{star}\left(O_{i}\right)$.

Given $l \in \Psi$ not meeting any $O_{i}$, then $l$ corresponds to an $\bar{l}$ in the second interpretation of $\Psi$ from centers $\bar{O}_{i}$. Let $\bar{P} \in \bar{l} \cap \bar{\pi}_{1}$. Then $l$ meets $\left(\bar{O}_{i} \bar{P}\right)^{\alpha_{i}^{-1}}(i=1,2,3)$, hence $l \in \Gamma_{\bar{*}_{1}}$.

"ᄃ". Let $l \in \Gamma_{\pi_{1}} \cap \Gamma_{\pi_{2}}$ and let $\bar{p}=\left(O_{1} l\right)^{\alpha_{1}} \cap\left(O_{2} l\right)^{\alpha_{2}}$.

First observe that homologous rays $r_{1}, r_{2}, r_{3}$ which meet $l$ must be of the form $r_{i}=$ $\left(\bar{O}_{i} \bar{P}\right)^{\alpha_{i}^{-1}}$ for some $\bar{P} \in \bar{p} \cap \bar{\pi}_{1} \cap \bar{\pi}_{2}$, because $r_{i}$ must be in $O_{i} l$, hence $r_{i}^{\alpha_{i}}$ must be in $\left(O_{i} l\right)^{\alpha_{i}}$, and $r_{i}^{\alpha_{i}}$ meets $\bar{p}(i=1,2)$. In particular, if $\bar{p} \cap \bar{\pi}_{1} \cap \bar{\pi}_{2}$ is a point, $r_{1}, r_{2}, r_{3}$ are unique.

Case 1. $\bar{p}$ meets $\bar{\pi}_{1} \cap \bar{\pi}_{2}$. Then either $\bar{p}=\bar{\pi}_{1} \cap \bar{\pi}_{2}$, whence $l \in \Delta$ by the observation above, or $\bar{p}$ does not lie in one of the planes, say $\bar{p} \nsubseteq \pi_{1}$. Then the intersection $\bar{p} \cap \bar{\pi}_{1} \cap \bar{\pi}_{2}$ is a point $\bar{P}$. Again by the observation above, $l$ must meet $\left(\bar{O}_{i} \bar{P}\right)^{\alpha_{i}^{-1}}$. Thus $l \in \Delta$.

Case 2. $\bar{p}$ does not meet $\bar{\pi}_{1} \cap \bar{\pi}_{2}$. Let $\bar{\pi}_{j} \cap \bar{p}=\bar{P}_{j}$. By the observation above, $\left(\bar{O}_{i} \bar{P}\right)^{\alpha_{i}^{-1}}$ meets $l$ for $i=1,2,3$ and $j=1,2$. In particular $\left(\bar{O}_{3} \bar{P}_{j}\right)^{\alpha_{3}^{-1}}$ meets $l$. But these two rays span $O_{i} l$. This means $\bar{O}_{i} \bar{p}$ are coaxial (with axis $\bar{p}$ ), and $O_{i} l=\left(\bar{O}_{i} \bar{P}\right)^{\alpha_{i}^{-1}}$ are coaxial with axis $l$. Thus $l \in \Psi$.

Corollary 5.1 If $\bar{\pi}_{1}, \bar{\pi}_{2}, \bar{\pi}_{3}, \bar{\pi}_{4}$ are planes in general position, then

$$
\Psi \cup \bigcup_{i=1,2,3} \operatorname{star}\left(O_{j}\right)=\bigcap_{i=1, \ldots, 4} \Gamma_{\hbar_{j}}
$$




\section{References}

1. Buchanan, $T .:$ On the critical set for photogrammetric reconstruction using line tokens in $\mathbf{P}_{3}(\mathbf{C})$. To appear.

2. Fano, G.: Studio di alcuni sistemi di rette considerati comme superficie dello spazio a cinque dimensioni. Ann. Mat., Ser. 2, 21 141-192 (1893).

3. Fano, G.: Nuove richerche sulle congruenze di rette del $3^{\circ}$ ordine prive di linea singolare. Mem. r. Acad. Sci. Torino, Ser. 2, 51, 1-79 (1902).

4. Hartshorne, R.: Algebraic Geometry. Berlin-Heidelberg-New York: Springer 1977.

5. Kliem, F.: Über Örter von Treffgeraden entsprechender Strahlen in eindeutig und linear verwandter Strahlengebilden erster bis vierter Stufe. Dissertation. Borna-Leipzig: Buchdruckerei Robert Noske 1909.

6. Krames, J.: Über die bei der Hauptaufgabe der Luftphotogrammetrie auftretende "gefährliche" Flächen. Bildmessung und Luftbildwesen (Beilage zur Allg. VermessungsNachr.) 17, Heft 1/2, 1-18 (1942).

7. Liu, Y., Huang, T.S.: Estimation of rigid body motion using straight line correspondences: further results. In: Proc. 8th Internat. Conf. Pattern Recognition (Paris 1986). Vol. I. pp. 306-309. Los Angeles, CA: IEEE Computer Society 1986.

8. Rinner, K., Burkhardt, R.: Photogrammetrie. In: Handbuch der Vermessungskunde. (Hsgb. Jordan, Eggert, Kneissel) Band III a/3. Stuttgart: J.B. Metzlersche Verlagsbuchhandlung 1972.

9. Roccella, D.: Sugli enti geometrici dello spazio di rette generate dalle intersezioni de' complessi corrispondenti in due o più fasci proiettivi di complessi lineari. Piazza Armerina: Stabilimento Tipografico Pansini 1882.

10. Schmid, T.: Über trilinear verwandte Felder als Raumbilder. Monatsh. Math. Phys. 6, 99106 (1895).

11. Semple, J.G., Kneebone, G.T.: Algebraic Projective Geometry. Oxford: Clarendon Press 1952, Reprinted 1979.

12. Severi, F.: Vorlesungen über Algebraische Geometrie. Geometrie auf einer Kurve, Riemannsche Flächen, Abelsche Integrale. Deutsche Übersetzung von E. Löffler. Leipzig-Berlin: Teubner 1921.

13. Sturm, R.: Die Lehre von den geometrischen Verwandtschaften. Leipzig - Berlin: B. G. Teubner 1909.

14. Walker, R.J.: Algebraic Curves. Princeton: University Press 1950. Reprint: New York: Dover 1962.

15. Zindler, K.: Algebraische Liniengeometrie. In: Encyklopädie der Mathematischen Wissenschaften. Leipzig: B.G. Teubner 1928. Band II, Teil 2, 2. Hälfte, Teilband A., pp. 973-1228. 